\title{
DEVELOPMENT OF DISTRIBUTED ION PUMPS FOR g-2 BEAM VACUUM SYSTEM*
}

\author{
H. C. Hseuh, M. Mapes and L. Snydstrup \\ AGS Department, Brookhaven National Laboratory \\ Associated Universities, Inc. \\ Upton, New York 11973
}

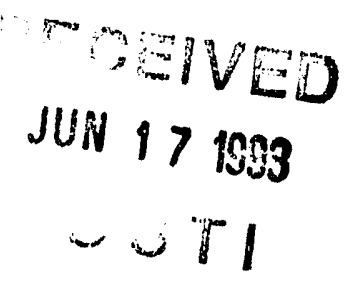

Abstract

Distributed ion pumps (DIPs) will be used for the beam vacuum system of the $g-2$ muon storage ring. The magnetic field intensity and alignment angle at the DIP locations are not uniform. The pumping behavior of several different ion pump elements under this non-uniform magnetic field has been studied. The results are compared with the theoretical predictions. Based on these results, the optimum design of the g-2 DIPs has been developed.

\section{INTRODUCTION}

For the precision measurement of the muon $\mathrm{g}-2$ value, a muon storage ring [1] is being constructed at Brookhaven. The principle equipment of the storage ring is the continuous superconducting magnet with $a \mathrm{~B}$ of $\approx 1.45$ Tesla and a diameter of $14 \mathrm{~m}$. Field homogeneity of $1 \mathrm{ppm}$ is required in the muon storage region which rules out the use of the conventional lumped ion pumps for the beam vacuum. DIPs have been used extensively in the bending magnet chambers of the electron storage rings as the main high vacuum pumps. With the existing bending field and the vacuum chambers, only Penning discharge cells are needed. In the g- 2 beam chambers, an area of $5 \mathrm{~cm}$ by $50 \mathrm{~cm}$ has been identified for the installation of the DIPs. The magnetic field at this location ranges from 12 to 15 kilogauss and the alignment angles (between the direction of B and the DIP anode axis) from $0^{\circ}$ to $9^{\circ}$. The pumping behavior of the Penning cells in this nonhomogeneous field has to be characterized before the implementation of the DIPs.

The pumping speed of the Penning cells is a function of $B$, the anode voltage $\mathrm{V}$ and the geometry of the cells. To prevent high voltage breakdown, $\mathrm{V}$ is usually limited to around 5 kilovolts. With the known $B$ and $V$, we can maximize the pumping speed $S$ by optimizing the geometry of the pump elements. Empirical formulae have been used by accelerator scientists to derive the optimum dimensions, such as the cell radius $R$, the cell length $L$ and the gaps, 8 , between the anodes and the cathode plates of the DIPs and to estimate the achievable $S$. The details of these formulae can be found in References 2, 3 and 4, and will not be repeated here.

*Work performed under the auspices of the U.S. Department of Energy.
Maleve [2] proposed the first practical formulae for deriving the geometry of the Penning cells. Based on his formulae, $S$ increases with increasing $B, R, L$ and $V$. Hartwig and Kouptsidis [3] pointed out later that $S$ peaks at a transition magnetic field, $B_{t r}$. They proposed different formulae for $S$ at $B$ below and above $B_{t r}$. For $B<B_{t r}$, $S$ is a function of $L, R, B$ and the pressure $P$. For $B \geq B_{t r}, S$ is independent of $R$ and $B$. However several recent studies $[5,6,7]$ indicated that $S$ increased with $R$ even at high $B$. To explain the dependence of $S$ on $R$ at high $B$, Suetsugu and Nakagawa [4] introduced the cell length and radius dependent total charge intensity $Q$ and anode sputtering efficiency $J$.

Good agreement with experimental data was achieved when $S$ was modified by $Q$ and $J$.

An additional variable for DIPs in the $\mathrm{g}-2$ chamber is the alignment angle $\boldsymbol{\theta}$. No systematic study of the dependence of $S$ on $\Theta$ in the Penning cells has been reported. Hartwig [3] suggested that the effect of the misalignment on the pumping speed can be estimated by replacing $R$ with the effective radius $R(\theta)(=R \cos \theta-0.5 \mathrm{~L} \sin \theta)$.

\section{DEVELOPMENT OF DIPs FOR g-2}

The DIP locations in the $g-2$ beam chambers has B ranging from 12 to 15 kilogauss and $\theta$ from $0^{\circ}$ to $9^{\circ}$. We have measured the pumping speeds of four ion pump elements at $B$ from a few hundred gauss to 15 kilogauss and $\theta$ from $0^{\circ}$ to $13^{\circ}$. The dimensions of the elements tested are listed in Table I. Elements $A 1$ and $A 2$ have the same $R$ and $L$ while $B$ and $C$ have larger cell radii. The results are summarized and compared with the predictions of the existing empirical formulae.

DIMENSIONS (in cm) OF THE ELEMENTS TESTED

\begin{tabular}{|c|c|c|c|}
\hline Elono, & R & 1 & \%colls \\
\hline Al & 0.6 & 1.9 & 92 \\
\hline$A 2$ & 0.6 & 1.9 & 140 \\
\hline B & 0.9 & 1.5 & 32 \\
\hline C & 1.2 & 2.5 & 57 \\
\hline
\end{tabular}

Table I. 
A bending magnet with a pole gap of $13 \mathrm{~cm}$ high, $25 \mathrm{~cm}$ wide and $120 \mathrm{~cm}$ long was used to supply the required B. A pumping speed measurement system was constructed for this study. It consists of a gas inlet chamber and a pumping chamber separated by an orifice with a conductance $C$ of $0.9 \mathrm{l} / \mathrm{sec}$ for nitrogen. The ion pump element was mounted in the pumping chamber centered in the magnet gap. Ion gauges were used to measure the pressures at both chambers. The pumping speed measurements were carried out at a pressure of low $10^{-7}$ Torr. Nitrogen was used as a test gas. It was bled into the gas chamber through a variable leak valve and pumped by the element through the orifice. The elements would pump gas at either 1.4 Tesla or 0.2 Tesla for a few hours before the measurement began. The magnet was then ramped to the desired $B$. The gauge readings were recorded after a steady state was reached at the selected $B$, which usually occurred in a few minutes. The above steps were then repeated for the next $B$. To test the effect of the alignment on the pumping speeds, the pumping chamber was tilted to an angle $\theta$ relative to the direction of $B$. The speed measurements were ther taken at this angle. Pumping speeds at the following angles were studied: $0^{\circ}, 3^{\circ}, 6^{\circ}, 9^{\circ}, 11^{\circ}$ and $13^{\circ}$. The pumping speeds were derived as $S=C^{*}\left(P_{g^{-}}\right.$ $\left.\mathrm{P}_{\text {pump }}\right) / \mathrm{P}_{\text {pump }}$.

The measured $S$ of each element at different $\Theta$ are plotted versus $B$ in Figure 1. As predicted by the empirical formulae, $S$ increased with increasing $B$ and peaked at $B_{t r}$. At $B \geq$ $B_{t r}$ when $\theta$ was small, $S$ of elements $A 1$ and $A 2$ remained constant with increasing $B$. At large $\Theta, S$ dropped off initially then levelled off. For elements B and C, S dropped off rapidly with increasing $B$ at all the angles. This dependence of $S$ on B for the large cells is not predicted by the empirical formulae. The calculated S for elements $A 1$ and $C$, using Suetsugu's modified formulae [4] are compared with the measured $S$ in Figure 2 for $\Theta=0^{\circ}$ and $9^{\circ}$. Reasonable agreement between the measured $S$ and the calculated $S$ was obtained for element A1.

The effects of alignment on $S$ are illustrated in Figure 3, where $S$ is plotted versus $Q$ for $B=0.2$ and 1.45 Tesla. The results were compared with the calculated results using Suetsugu's formulae and $R(\theta)$. Reasonable agreement between the calculated $S$ and the measured $S$ was obtained for
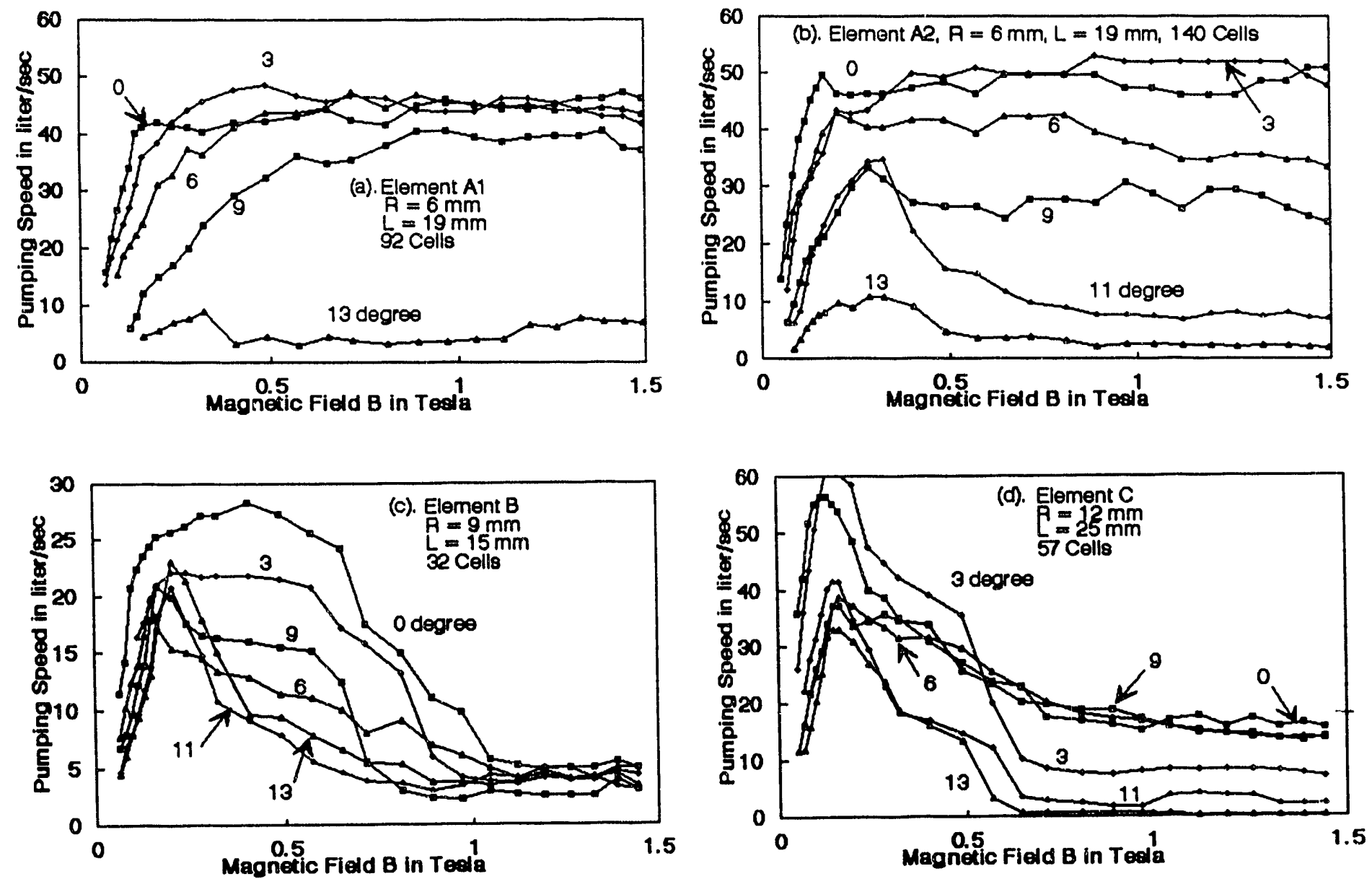

Figure 1. Measured pumping speeds of several ion pump elements as a function of the magnetic field. The numbers above are the alignment angles. 
element $\mathrm{Al}$ at 1.45 Tesla; and for elements $\mathrm{B}$ and $\mathrm{C}$ at 0.2 Tesla.
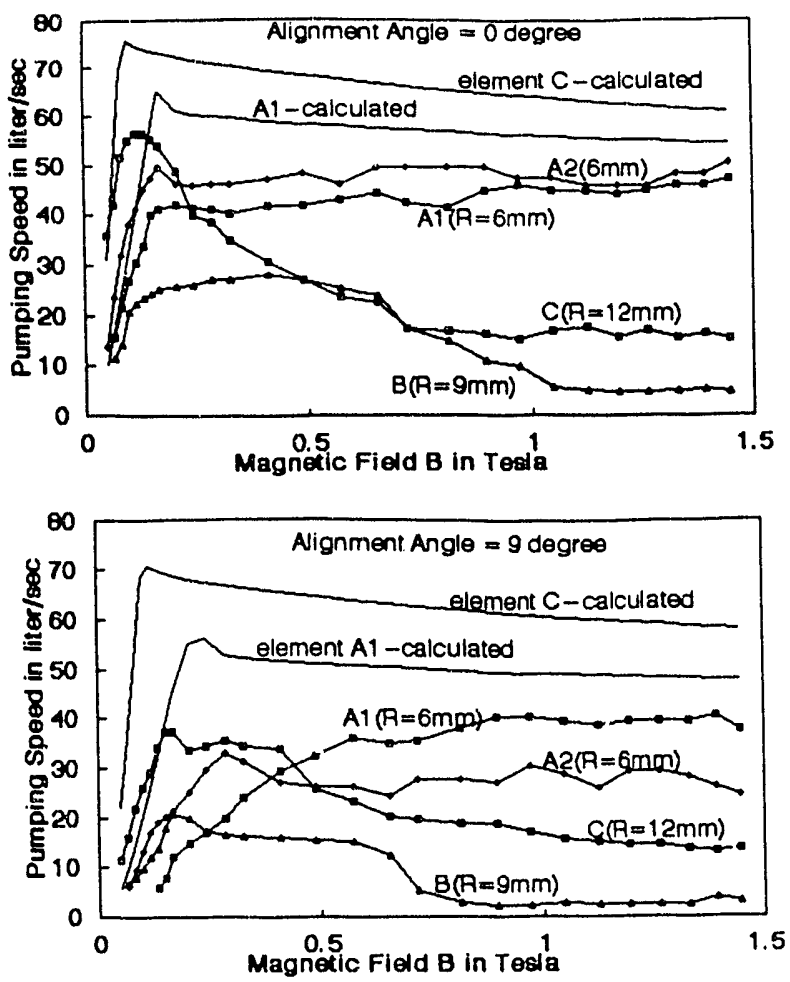

Fig. 2. Comparison between the calculated pumping speeds and the measured pumping speeds at alignment angles of $0^{\circ}$ and $9^{\circ}$

In summary, at high B, the measured pumping speeds of the elements of small cells are substantially higher than those of the elements of large cells even at $9^{\circ}$. These results are in reasonable agreement with the calculated results. The pumping speeds of the elements of large cells decreased rapidly with increasing $B$, which are not predicted by the existing empirical formulae. At high B, the effect of misalignment on the pumping speeds of elements with small cells is in reasonable agreement with those calculated using $R(\theta)$.

\section{OPTIMUM DIP DESIGN FOR $\mathrm{g}-2$}

The results of our study indicate that the pumping speed of the elements with $R \geq 9 \mathrm{~mm}$ dropped off rapidly with increasing $B$ and $\boldsymbol{\theta}$, which sets the upper bound for the cell radius of the g-2 DIPs. The minimum cell radius can be estimated from $P_{\min }$, the minimum operating pressure as defined by Maleve [2]. At a $P_{\min }$ of $1 * 10^{-8}$ Torr, the smallest radius of the pseudo cells (the space between the regular cells that gives extra pumping) should be $1.5 \mathrm{~mm}$ and that of the regular cells $4 \mathrm{~mm}$. Other measurements $[5,6,7]$ at high B gave a minimum radius of $5 \mathrm{~mm}$, below that the pumping speed dropped off. This is consistent with the fact that the total discharge intensity $Q$ [4] decreases with $R$.
Taking all these facts into consideration, the optimum cell radius seems to be around $6 \mathrm{~mm}$.
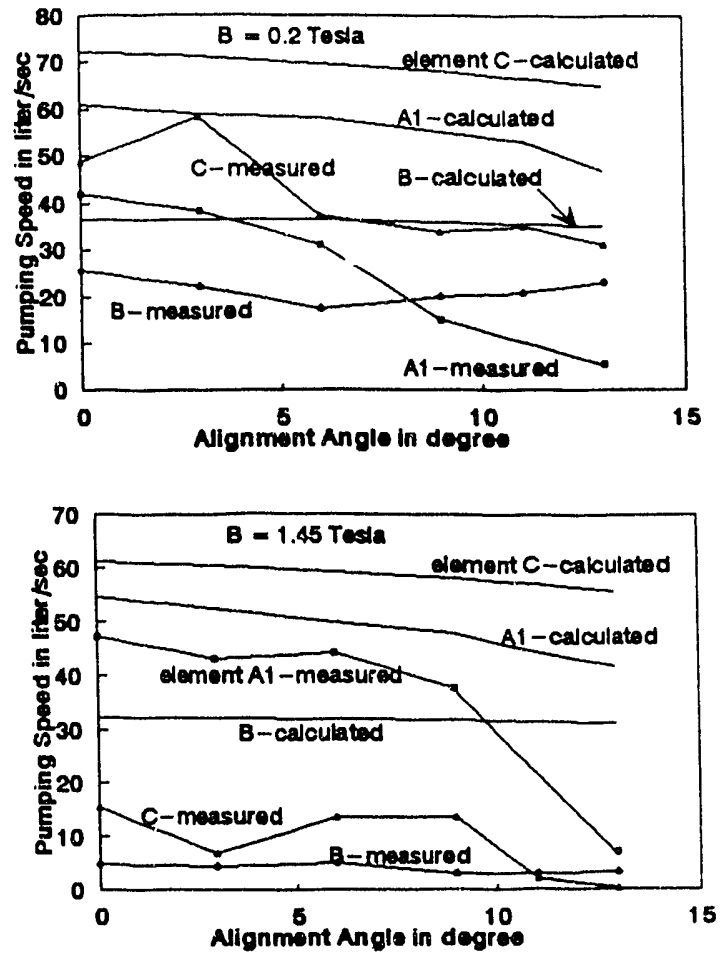

Fig. 3. The dependence of the pumping speeds on the alignment angle $\theta$ at $\mathrm{B}=0.2 \mathrm{~T}$ and $1.45 \mathrm{~T}$ for elements $\mathrm{Al}, \mathrm{B}$, and $\mathrm{C}$.

To limit the pumping speed loss due to the conductance restriction to less than 10\%, Maleve [2] proposed that the cell length $L$ should be between 3-4 times of $R$, or around $20 \mathrm{~mm}$. The gaps $\delta$ between the cells and the cathode plates must be reasonably large for conductance purposes and to prevent high voltage breakdown. Gaps of $5 \mathrm{~mm}$ will meet these requirements.

A totai of $\mathbf{2 5 0}$ cells of the above geometry in three layers can be packed at each g-2 DIP location. Based on Suetsugu's formulae, as well as the performance of elements A1 and A2, pumping speed of over $100 \mathrm{l} / \mathrm{sec}$ can be realized from each g-2 DIP.

\section{REFERENCES}

[1] V.W. Hughes, in Particle, Strings \& Cosmology, World Scientist, Singapore, p.868, 1992.

[2] M.D. Maleve and E.M. Trachtenberg, Vacuum, 23, 403 (1973).

[3] H. Hartwig and J.S.Kouptsidis, J. Vac. Sci. Technol., 11,1154 (1974).

[4] Y.Suetsugu and M. Nakagawa, Vacuum, 42, 761 (1991).

[5] T.S. Chou, J. Vac. Sci. Technol., A5, 3446 (1987).

[6] Y.Suetsugu and M. Nakagawa, Vacuum, 42, 625 (1991).

[7] B.A. Trickett, Vacuum, 28, 471 (1978). 

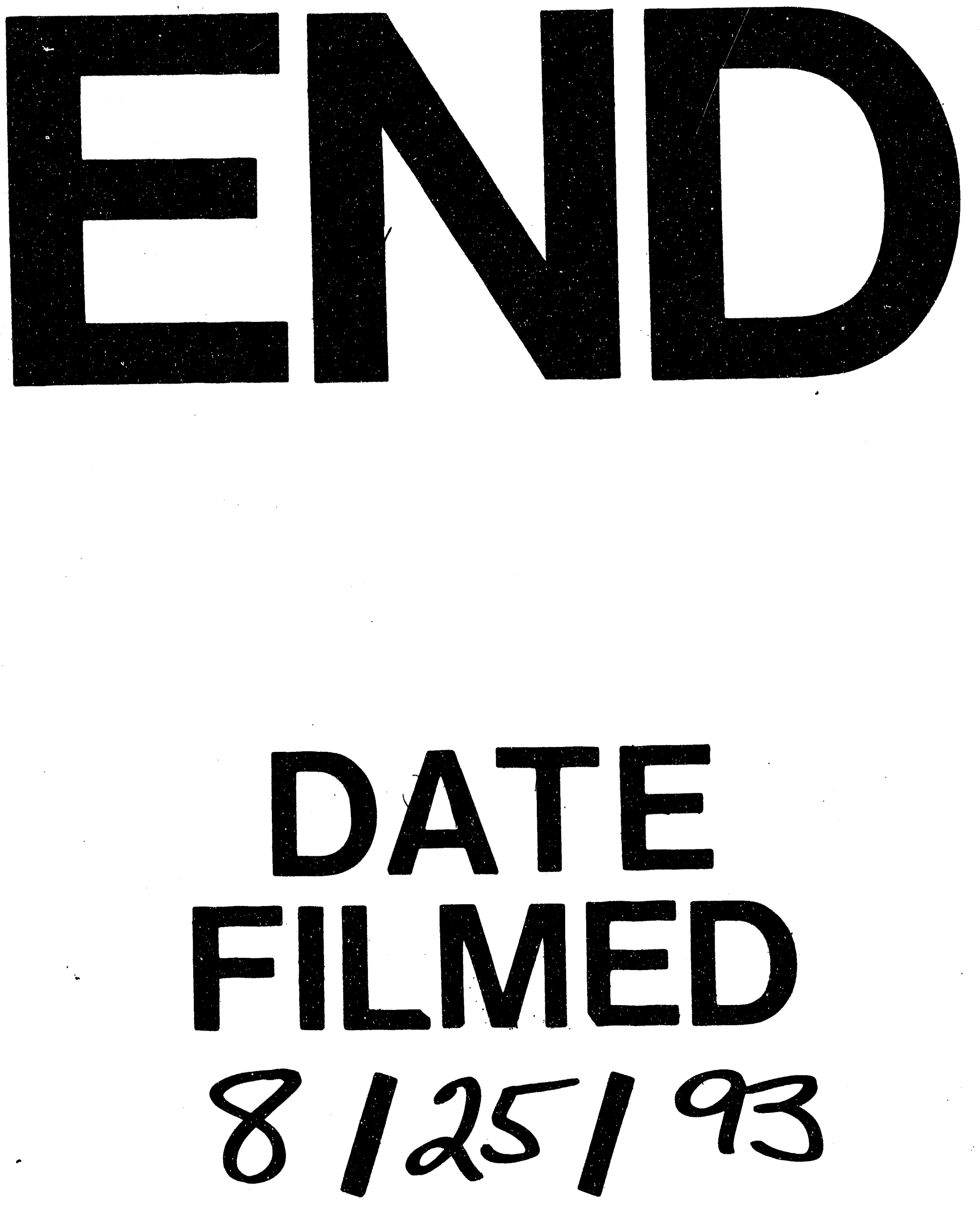

1 
? 